\title{
Chiral Brønsted Acid-Catalyzed Inverse Electron-demand Aza Diels-Alder Reaction
}

\author{
Takahiko Akiyama,* Hisashi Morita, and Kohei Fuchibe \\ Department of Chemistry, Faculty of Science, \\ Gakushuin University, \\ Mejiro, Toshima-ku, Tokyo 171-8588, Japan \\ takahiko.akiyama@gakushuin.ac.jp
}

\section{Supporting Information}

General. NMR spectra were recorded on Unity Inova-400 instrument (Varian Inc., 400 $\mathrm{MHz}$ for ${ }^{1} \mathrm{H}, 100 \mathrm{MHz}$ for ${ }^{13} \mathrm{C}, 189 \mathrm{MHz}$ for ${ }^{31} \mathrm{P}$ ) and JNM-A1300 instrument (JEOL, 300 $\mathrm{MHz}$ for ${ }^{1} \mathrm{H}, 75 \mathrm{MHz}$ for ${ }^{13} \mathrm{C}, 121 \mathrm{MHz}$ for ${ }^{31} \mathrm{P}, 283 \mathrm{MHz}$ for ${ }^{19} \mathrm{~F}$ ) using $\mathrm{CDCl}_{3}$ as a solvent. Tetramethylsilane (TMS) $(\delta=0)$ or $\mathrm{CHCl}_{3}(\delta=7.27)$ served as an internal standard for ${ }^{1} \mathrm{H} \mathrm{NMR}$, and $\mathrm{CDCl}_{3}$ was used as an internal standard $(\delta=77.0)$ for ${ }^{13} \mathrm{C}$ NMR. $\quad \mathrm{H}_{3} \mathrm{PO}_{4}$ was used as an external standard $(\delta=0)$ for ${ }^{31} \mathrm{P}$ NMR. $\mathrm{C}_{6} \mathrm{~F}_{6}$ was used as an internal standard $(\delta=0)$ for ${ }^{19} \mathrm{~F}$ NMR. IR spectra were recorded on a Shimadzu FT-IR 8600 spectrometer. EI Mass spectra were measured by GCMS-QP-5000 (Shimadzu) at an ionizing voltage of $70 \mathrm{eV}$. Fab mass spectra were measured on a JEOL JMS-700 using 3-nitrobenzyl alcohol as a matrix. Purification of the products was performed by column chromatography on silica gel (Fuji sylisia D60L or PSQ-60B) or preparative TLC on silica gel (Wako gel B-5F). All solvents were purified according to the standard procedures. Elemental analysis (EA) was carried out on EA1110 instrument (Amco Inc.).

\section{Determination of the absolute stereochemistry}

The absolute and relative stereochemistry of the following 2 compounds were determined by X-ray analysis. 
<smiles>CCOC1C[C@@H](c2ccc(Br)cc2)Nc2c(O)cccc21</smiles>

This compound ( $90 \%$ ee, Table 1, entry 6) was recrystallized from ethyl acetate to give optically pure compound, which was subjected to X-ray analysis (see, quinoline1.cif).
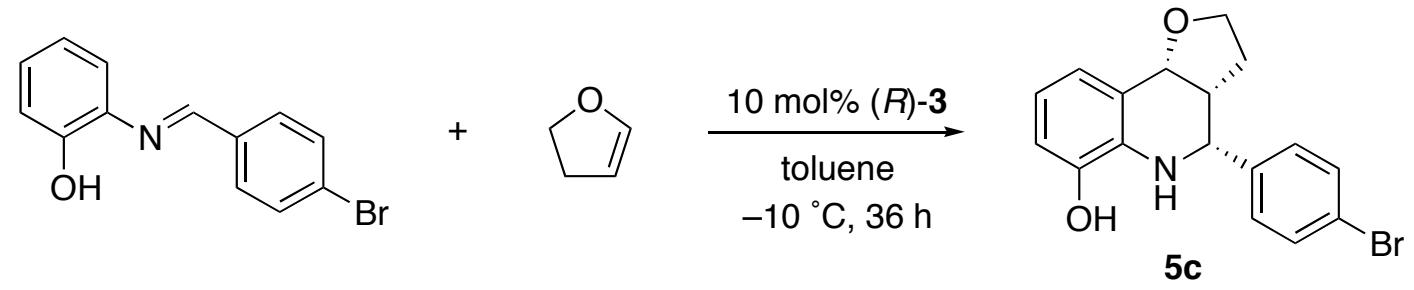

$68 \%$, cis:trans $=99: 1$

$73 \%$ ee $(c i s)$

The cycloadduct 5c was recrystallized from ethanol to give optically pure compound, which was subjected to X-ray analysis (see, quinoline2.cif).

\section{Experimental Section}<smiles>O=P(O)(O)Oc1c(-c2c3ccccc3cc3ccccc23)cc2ccccc2c1-c1c(-c2c3ccccc3cc3ccccc23)cc2ccccc2c1O</smiles>

$[\alpha]_{\mathrm{D}}{ }^{26}-24.9$ (c 1.00, EtOH); m.p. $>300{ }^{\circ} \mathrm{C}(\mathrm{EtOH}) ; \mathrm{IR}\left(\mathrm{CHCl}_{3}\right) 3057,3011,1601,1497$, 1445, 1425, 1402, 1356, 1319, 1265, 1232, 1207, 1151, 1103, 1088, 1015, 972, 949, 887, 872, 860, $845 \mathrm{~cm}^{-1} ;{ }^{1} \mathrm{H}$ NMR (400 MHz, $\left.\mathrm{CDCl}_{3}\right) \delta=8.10(\mathrm{~s}, 2 \mathrm{H}), 7.98(\mathrm{~s}, 2 \mathrm{H})$, 7.96 (d, $2 \mathrm{H}, J=8.2 \mathrm{~Hz}), 7.81$ (d, $2 \mathrm{H}, J=8.4 \mathrm{~Hz}), 7.68$ (d, $1 \mathrm{H}, J=8.4 \mathrm{~Hz}), 7.64-7.45$ (m, $10 \mathrm{H})$, 7.35-7.31 (m, $2 \mathrm{H}), 7.25-7.21(\mathrm{~m}, 2 \mathrm{H}), 7.09-7.00$ (m, $4 \mathrm{H}), 3.66$ (brs, $1 \mathrm{H}) ;{ }^{13} \mathrm{C}$ $\operatorname{NMR}\left(75 \mathrm{MHz}, \mathrm{CDCl}_{3}\right) \delta=146.6,146.5,133.8,132.7,131.3,131.2,131.1,130.8,130.7$, $130.3,128.5,127.9,127.6,127.4,127.3,126.9,126.2$, 126.0, 125.9, 124.9, 124.9, 124.8, 
122.4, 122.4; ${ }^{31} \mathrm{P}$ NMR (121 MHz, $\left.\mathrm{CDCl}_{3}\right) \delta=0.61$; $\mathrm{MS}(\mathrm{Fab}) \mathrm{m} / \mathrm{z} 701[\mathrm{M}+1]^{+}$; Found: $\mathrm{C}$, 82.07; H, 4.16\%. Calcd for $\mathrm{C}_{48} \mathrm{H}_{29} \mathrm{O}_{4} \mathrm{P}: \mathrm{C}, 82.28 ; \mathrm{H}, 4.17 \%$.

General procedure for the aza Diels-Alder reaction (entry 1, Table 1 is shown) To a solution of $N$-benzylidene-2-aminopheol (68.4 $\mathrm{mg}, 0.347 \mathrm{mmol})$ and (R)-3,3'-bis(9-anthryl)-1,1'-binaphthyl phosphate $(\boldsymbol{R})-3$ (24.3 $\mathrm{mg}, 0.0347 \mathrm{mmol})$ in toluene $(0.5 \mathrm{~mL})$ was added ethyl vinyl ether $(50 \mu 1,0.52 \mathrm{mmol})$ at $-10{ }^{\circ} \mathrm{C}$. Being stirred at the temperature for $44 \mathrm{~h}$ by confirming the disappearance of the imine by TLC, the mixture was quenched by addition of sat. $\mathrm{NaHCO}_{3}$ solution. The solution was extracted with ethyl acetate. The combined organic layers were successively washed with $1 \mathrm{~N} \mathrm{HCl}$, brine, dried over anhydrous $\mathrm{Na}_{2} \mathrm{SO}_{4}$, and concentrated to dryness. The crude product was purified by preparative TLC on silica gel (hexane/EtOAc $=4 / 1)$ to give the product $(84.1 \mathrm{mg}, 0.31 \mathrm{mmol})$ in $89 \%$ yield and with $94 \%$ ee, which was determined by chiral HPLC analysis on a Daicel Chiralcel OD-H column.

\section{4-Ethoxy-2-phenyl-1,2,3,4-tetrahydroquinolin-8-ol}<smiles>CCOC1C[C@@H](c2ccccc2)Nc2c(O)cccc21</smiles>

$\mathrm{R}_{\mathrm{f}} 0.4($ Hexane : Ethyl acetate $=4: 1 \mathrm{v} / \mathrm{v})$; amorphous solid; $[\alpha]_{\mathrm{D}}{ }^{25} 25.0\left(\mathrm{c} 1.1, \mathrm{CHCl}_{3}\right)$ (94\% ee); ${ }^{1} \mathrm{H}$ NMR (400 MHz, $\left.\mathrm{CDCl}_{3}\right) \delta=7.46-7.28(\mathrm{~m}, 5 \mathrm{H}), 7.08-6.97(\mathrm{~m}, 1 \mathrm{H})$, 6.63-6.50 (m, 2H), 5.28 (brs, 1H), 4.86 (d, 1H, $J=10.2,5.7 \mathrm{~Hz}), 4.50$ (brd, 1H, $J=11.8$ $\mathrm{Hz}), 4.28$ (brs, 1H), 3.72-3.63 (m, 1H), 3.61-3.53 (m, 1H), 2.43-2.36 (m, 1H) 2.16-2.04 $(\mathrm{m}, 1 \mathrm{H}), 1.22(\mathrm{t}, 3 \mathrm{H}, J=7.0 \mathrm{~Hz}) ;{ }^{13} \mathrm{C}$ NMR $\left(75 \mathrm{MHz}, \mathrm{CDCl}_{3}\right) \delta=143.7,142.1,134.0$, $128.6,127.7,126.7,123.6,119.66,116.9,113.5,74.1,63.4,36.9,25.3,15.6$; IR $\left(\mathrm{CHCl}_{3}\right)$ 3061, 3414, 3290, 2995, 2856, 1726, 1620, 1597, 1497, 1377, 1331, 1269, 1153, $1072 \mathrm{~cm}^{-1}$; Found: C, 75.62; H, 7.16; N, 5.04\%. Calcd for $\mathrm{C}_{17} \mathrm{H}_{19} \mathrm{NO}_{2}: \mathrm{C}, 75.81$; H, 7.11; N, 5.20\%; HPLC: Daicel Chiralcel OJ-H, Hexane $/ i-\mathrm{PrOH}=5 / 1$, Flow rate $=0.5$ $\mathrm{mL} / \mathrm{min}, \mathrm{UV}=254 \mathrm{~nm}, \mathrm{t}_{\mathrm{R}}=38.4 \min (2 R, 4 R), \mathrm{t}_{\mathrm{R}}=30.3 \min (2 S, 4 S)$. 


\section{2-(4-Chlorophenyl)-4-ethoxy-1,2,3,4-tetrahydroquinolin-8-ol}<smiles>CCOC1C[C@@H](c2ccc(Cl)cc2)Nc2c(O)cccc21</smiles>

$\mathrm{R}_{\mathrm{f}} 0.4$ (Hexane: Ethyl acetate $\left.=4: 1\right)$; amorphous solid; $[\alpha]_{\mathrm{D}}{ }^{25} 31.0$ (c 1.1, $\left.\mathrm{CHCl}_{3}\right)(88 \%$ ee); IR $\left(\mathrm{CHCl}_{3}\right)$ 3601, 3398, 3294, 3007, 2980, 2862, 1616, 1593, 1493, 1445, 1331, 1269, 1242, 1096, $1015 \mathrm{~cm}^{-1}$; ${ }^{1} \mathrm{H}$ NMR (400 MHz, $\left.\mathrm{CDCl}_{3}\right) \delta=7.38$ (d, 2H, $\left.J=8.4 \mathrm{~Hz}\right)$, $7.31(\mathrm{~d}, 2 \mathrm{H}, J=8.4 \mathrm{~Hz}), 7.02-6.95(\mathrm{~m}, 1 \mathrm{H}), 6.62-6.54(\mathrm{~m}, 2 \mathrm{H}), 5.18$ (brs, 1H), 4.83 (dd, $1 \mathrm{H}, J=11.2,5.5 \mathrm{~Hz}$ ), 4.48 (brd, 1H, $J=11.2 \mathrm{~Hz}), 4.25$ (brs, 1H), 3.72-3.50 (m, 2H), 2.38-2.32 (m, 1H), 2.07 (ddd, $1 \mathrm{H}, J=11.2,11.2,11.2 \mathrm{~Hz}), 1.21(\mathrm{t}, 3 \mathrm{H}, J=7.0 \mathrm{~Hz}) ;{ }^{13} \mathrm{C}$ $\operatorname{NMR}\left(75 \mathrm{MHz}, \mathrm{CDCl}_{3}\right) \delta=142.2,141.9,133.7,133.2,128.7,128.0,123.5,119.8,119.8$, 117.1, 113.7, 73.9, 63.5, 36.8, 15.6; Found: C, 67.19; H, 5.94; N, 4.54\%. Calcd for $\mathrm{C}_{17} \mathrm{H}_{18} \mathrm{ClNO}_{2}$ : C, 67.21; H, 5.97; N, 4.61\%; HPLC: Daicel Chiralcel OJ-H, Hexane $/ \mathrm{EtOH}=5 / 1$, Flow rate $=0.5 \mathrm{~mL} / \mathrm{min}, \mathrm{UV}=254 \mathrm{~nm}, \mathrm{t}_{\mathrm{R}}=25.0 \min (2 R, 4 R), \mathrm{t}_{\mathrm{R}}=$ $20.4 \min (2 S, 4 S)$.

\section{2-(4-Bromophenyl)-4-ethoxy-1,2,3,4-tetrahydroquinolin-8-ol}<smiles>CCOC1C[C@@H](c2ccc(Br)cc2)Nc2c(O)cccc21</smiles>

$\mathrm{R}_{\mathrm{f}} 0.4$ (Hexane: Ethyl acetate $\left.=4: 1\right) ; \mathrm{mp} 144.0-145.0{ }^{\circ} \mathrm{C}$ (toluene); $[\alpha]_{\mathrm{D}}{ }^{26} 26.8(\mathrm{c} 1.0$, $\left.\mathrm{CHCl}_{3}\right)\left(90 \%\right.$ ee); IR $\left(\mathrm{CHCl}_{3}\right) 3601,2285,2999,2980,2862,1620,1593,1489,1447$, 1333, 1269, 1246, 1177, 1157, 1103, 1073, $957 \mathrm{~cm}^{-1}$; ${ }^{1} \mathrm{H}$ NMR (400 MHz, $\mathrm{CDCl}_{3}$ ) $\delta=7.48(\mathrm{~d}, 2 \mathrm{H}, J=8.1 \mathrm{~Hz}), 7.35(\mathrm{~d}, 2 \mathrm{H}, J=8.1 \mathrm{~Hz}), 7.06-7.00(\mathrm{~m}, 1 \mathrm{H}), 6.66-6.57(\mathrm{~m}, 2 \mathrm{H})$, 4.82 (dd, 1H, $J=10.6,6.0 \mathrm{~Hz}$ ), 4.57 (brs, 1H), 4.5 (brd, 1H, $J=8.4 \mathrm{~Hz}$ ), 4.22 (brs, $1 \mathrm{H}$ ), 3.71-3.62 (m, 1H), 3.49-3.51 (m, 1H), 2.42-2.34 (m, 1H), 2.07 (dd, 1H, J=10.6, 10.6 $\mathrm{Hz}), 1.23(\mathrm{t}, 3 \mathrm{H}, J=7.0 \mathrm{~Hz}) ;{ }^{13} \mathrm{C} \mathrm{NMR}\left(75 \mathrm{MHz}, \mathrm{CDCl}_{3}\right) \delta=142.8,141.8,133.6,131.7$, 128.4, 123.6, 121.3, 119.9, 117.1, 73.8, 63.5, 55.0, 36.8, 15.6; Found: C, 58.89; H, 5.30; N, 4.04\%. Calcd for $\mathrm{C}_{17} \mathrm{H}_{18} \mathrm{BrNO}_{2}$ : C, 58.63; H, 5.21; N, 4.02\%; HPLC: Daicel Chiralcel OJ-H, Hexane $/ \mathrm{EtOH}=5 / 1$, Flow rate $=0.5 \mathrm{~mL} / \mathrm{min}, \mathrm{UV}=254 \mathrm{~nm}, \mathrm{t}_{\mathrm{R}}=20.7$ 
$\min (2 R, 4 R), \mathrm{t}_{\mathrm{R}}=16.0 \min (2 S, 4 S)$.

\section{4-Ethoxy-2-(4-trifluoromethylphenyl)-1,2,3,4-tetrahydroquinolin-8-ol}<smiles>CCOC1C[C@@H](c2ccc(C(F)(F)F)cc2)Nc2c(O)cccc21</smiles>

$\mathrm{R}_{\mathrm{f}} 0.4$ (Hexane: Ethyl acetate $\left.=4: 1\right)$; amorphous solid; $[\alpha]_{\mathrm{D}}{ }^{25} 32.5$ (c 1.0, $\left.\mathrm{CHCl}_{3}\right)(84 \%$ ee); IR $\left(\mathrm{CHCl}_{3}\right) 3595,3393,3294,3013,2974,2928,2851,1620,1593,1327,1269$, $1246,1165,1128,1070,1015 \mathrm{~cm}^{-1} ;{ }^{1} \mathrm{H}$ NMR $\left(400 \mathrm{MHz}, \mathrm{CDCl}_{3}\right) \delta=7.59(\mathrm{~d}, 2 \mathrm{H}$, $J=8.4 \mathrm{~Hz}), 7.55(\mathrm{~d}, 2 \mathrm{H}, J=8.4 \mathrm{~Hz}), 7.06-6.95(\mathrm{~m}, 1 \mathrm{H}), 6.68-6.52(\mathrm{~m}, 2 \mathrm{H}), 5.41$ (brs, $1 \mathrm{H})$, 4.88-4.79 (m, 1H), 4.63-4.55 (m, 1H), 4.30 (brs, 1H), 3.68-3.51 (m, 2H), 2.42-2.35 (m, 1H) $2.13(\mathrm{ddd}, 1 \mathrm{H}, J=10.6,10.6,10.6 \mathrm{~Hz}), 1.24(\mathrm{t}, 3 \mathrm{H}, J=7.1 \mathrm{~Hz}) ;{ }^{13} \mathrm{C} \mathrm{NMR}(75 \mathrm{MHz}$, $\left.\mathrm{CDCl}_{3}\right) \delta=147.9,142.0,133.5,129.8$ (q, $\left.J=31.8 \mathrm{~Hz}\right), 127.0,125.5,123.2,122.3,119.8$, 117.2, 113.7, 73.9, 63.5, 55.1, 36.6, 15.4.; ${ }^{19} \mathrm{~F}-\mathrm{NMR}\left(282 \mathrm{MHz}, \mathrm{CDCl}_{3}\right) \delta \quad 0.19$; Found: $\mathrm{C}, 63.97 ; \mathrm{H}, 5.54 ; \mathrm{N}, 4.04 \%$. Calcd for $\mathrm{C}_{18} \mathrm{H}_{18} \mathrm{~F}_{3} \mathrm{NO}_{2}: \mathrm{C}, 64.09 ; \mathrm{H}, 5.38 ; \mathrm{N}$, 4.15\%; HPLC: Daicel Chiralcel OJ-H, Hexane $/ i-\mathrm{PrOH}=10 / 1$, Flow rate $=0.5$ $\mathrm{mL} / \mathrm{min}, \mathrm{UV}=254 \mathrm{~nm}, \mathrm{t}_{\mathrm{R}}=40.6 \min (2 R, 4 R), \mathrm{t}_{\mathrm{R}}=32.3 \min (2 S, 4 S)$.

\section{4-Ethoxy-2-(4-trimethylphenyl)-1,2,3,4-tetrahydroquinolin-8-ol}<smiles>CCO[C@H]1C[C@@H](c2ccc(C)cc2)Nc2c(O)cccc21</smiles>

$\mathrm{R}_{\mathrm{f}} 0.3$ (Hexane: Ethyl acetate $\left.=4: 1\right)$; amorphous solid; $[\alpha]_{\mathrm{D}}{ }^{27} 20.0$ (c 1.0, $\left.\mathrm{CHCl}_{3}\right)(91 \%$ ee); IR $\left(\mathrm{CHCl}_{3}\right)$ 3601, 3398, 3308, 3007, 2974, 2928, 2862, 1616, 1593, 1497, 1445, 1327, 1271, 1246, 1155, 1072, $1057 \mathrm{~cm}^{-1} ;{ }^{1} \mathrm{H}$ NMR (400 MHz, $\left.\mathrm{CDCl}_{3}\right) \delta=7.34(\mathrm{~d}, 2 \mathrm{H}$, $J=7.9 \mathrm{~Hz}), 7.16(\mathrm{~d}, 2 \mathrm{H}, J=7.9 \mathrm{~Hz}), 7.07-6.96(\mathrm{~m}, 1 \mathrm{H}), 6.65-6.49(\mathrm{~m}, 2 \mathrm{H}), 5.11$ (brs, 1H), $4.86(\mathrm{dd}, 1 \mathrm{H}, J=10.6,5.7 \mathrm{~Hz}), 4.45$ (brd, $1 \mathrm{H}, J=10.8 \mathrm{~Hz}), 3.73-3.63$ (m, 1H), 3.61-3.53 (m, 1H), 2.42-2.30 (m, 1H), 2.35 (s, 3H), 2.09 (ddd, 1H, $J=12.1,10.8,10.6 \mathrm{~Hz}), 1.25$ (t, $3 \mathrm{H}, J=7.0 \mathrm{~Hz}) ;{ }^{13} \mathrm{C}$ NMR $\left(75 \mathrm{MHz}, \mathrm{CDCl}_{3}\right) \delta=142.0,140.7,137.4,134.1,129.3$, 126.6, 123.6, 119.6, 116.9, 113.6, 74.2, 63.4, 55.5, 37.0, 21.1, 15.6; Found: C, 76.33; $\mathrm{H}, 7.26 ; \mathrm{N}, 4.92 \%$. Calcd for $\mathrm{C}_{18} \mathrm{H}_{21} \mathrm{NO}_{2}: \mathrm{C}, 76.29 ; \mathrm{H}, 7.47 ; \mathrm{N}, 4.94 \%$; HPLC: 
Daicel Chiralcel OJ-H, Hexane $/ \mathrm{EtOH}=15 / 1$, Flow rate $=0.5 \mathrm{~mL} / \mathrm{min}, \mathrm{UV}=254 \mathrm{~nm}$, $\mathrm{t}_{\mathrm{R}}=52.6 \min (2 R, 4 R), \mathrm{t}_{\mathrm{R}}=49.2 \min (2 S, 4 S)$

\section{2-(2-Chlorophenyl)-4-ethoxy-1,2,3,4-tetrahydroquinolin-8-ol}<smiles>CCOC1C[C@@H](c2ccccc2Cl)Nc2c(O)cccc21</smiles>

$\mathrm{R}_{\mathrm{f}} 0.4$ (Hexane: Ethyl acetate $=4: 1$ ); amorphous solid; $[\alpha]_{\mathrm{D}}{ }^{26} 62.3\left(\mathrm{c} 0.6, \mathrm{CHCl}_{3}\right)$ (87\% ee); IR $\left(\mathrm{CHCl}_{3}\right)$ 3599, 3402, 3302, 3007, 2986, 2937, 2874, 1730, 1620, 1591, 1497, 1441, 1334, 1265, 1248, 1153, 1103, 1074, $1055 \mathrm{~cm}^{-1} ;{ }^{1} \mathrm{H}$ NMR (400 MHz, $\left.\mathrm{CDCl}_{3}\right) \delta=7.73-7.68(\mathrm{~m}, 1 \mathrm{H}), 7.40-7.17(\mathrm{~m}, 3 \mathrm{H}), 7.10-6.96(\mathrm{~m}, 1 \mathrm{H}), 6.70-6.48(\mathrm{~m}, 2 \mathrm{H})$, 5.21 (brs, 1H), 5.00 (brd, 1H, J=9.5 Hz), 4.86-4.82 (m, 1H), 4.25 (brs, 1H), 3.68-3.51 $(\mathrm{m}, 2 \mathrm{H}), 2.51-2.46(\mathrm{~m}, 1 \mathrm{H}), 2.11-2.02(\mathrm{~m}, 1 \mathrm{H}), 1.19(\mathrm{t}, 3 \mathrm{H}, J=7.0 \mathrm{~Hz}) ;{ }^{13} \mathrm{C}$ NMR $(75$ $\left.\mathrm{MHz}, \mathrm{CDCl}_{3}\right) \delta=142.1,140.9,133.9,132.4,129.4,128.4,127.9,127.3,23.5,120.1$, 117.0, 113.6, 73.8, 63.5, 51.4, 34.3, 15.5; Found: C, 67.09; H, 6.03; N, 4.41\%. Calcd for $\mathrm{C}_{17} \mathrm{H}_{18} \mathrm{ClNO}_{2}$ : C, 67.21; H, 5.97; N, 4.61\%; HPLC: Daicel Chiralcel OJ-H, Hexane $/ \mathrm{EtOH}=5 / 1$, Flow rate $=0.5 \mathrm{~mL} / \mathrm{min}, \mathrm{UV}=254 \mathrm{~nm}, \mathrm{t}_{\mathrm{R}}=24.5 \mathrm{~min}(2 R, 4 R), \mathrm{t}_{\mathrm{R}}=20.2$ $\min (2 S, 4 S)$.

\section{4-Ethoxy-2-naphthyl-1,2,3,4-tetrahydroquinolin-8-ol}<smiles>CCOC1C[C@@H](c2ccc3ccccc3c2)Nc2c(O)cccc21</smiles>

$\mathrm{R}_{\mathrm{f}} 0.4$ (Hexane: Ethyl acetate=4:1); amorphous solid; $[\alpha]_{\mathrm{D}}{ }^{26} 34.3$ (c $\left.1.15, \mathrm{CHCl}_{3}\right)(87 \%$ ee); IR $\left(\mathrm{CHCl}_{3}\right)$ 3601, 3398, 3288, 2999, 2980, 2862, 1620, 1587, 1441, 1373, 1271, 1242, 1151, 1105, 1076, $1057 \mathrm{~cm}^{-1}$; ${ }^{1} \mathrm{H}$ NMR (400 MHz, $\left.\mathrm{CDCl}_{3}\right) \delta=7.91(\mathrm{~s}, 1 \mathrm{H})$, 7.88-7.81 (m, 3H), 7.59 (d, 1H, J=7.1 Hz), 7.52-7.43 (m, 2H), 7.08 (brs, 1H), 6.62 (brs, 2H), 4.91 (brs, 1H), 4.82 (brs, 1H), 4.69 (brs, 1H), 4.34 (brs, 1H), 3.76-3.65 (m, 1H), 3.64-3.54 (m, 1H), 2.54-2.42 (m, 1H), 2.20 (ddd, $1 \mathrm{H}, J=11.5,11.5,11.5 \mathrm{~Hz}), 1.25(\mathrm{t}, 3 \mathrm{H}$, $J=7.0 \mathrm{~Hz}) ;{ }^{13} \mathrm{C}$ NMR $\left(75 \mathrm{MHz}, \mathrm{CDCl}_{3}\right) \delta=142.1,140.9,133.4,133.1,128.4,127.8$, $127.6,126.1,125.8,125.3,124.8,119.5,117.0,113.7,74.6,63.4,55.8,36.8,15.6$; 
Found: C, 78.67; H, 6.57; N, 4.44\%. Calcd for $\mathrm{C}_{21} \mathrm{H}_{21} \mathrm{NO}_{2}$ : C, 78.97; H, 6.63; N, 4.39\%. HPLC: Daicel Chiralcel OJ-H, Hexane/EtOH=5/1, Flow rate $=0.5 \mathrm{~mL} / \mathrm{min}, \mathrm{UV}=254 \mathrm{~nm}$, $\mathrm{t}_{\mathrm{R}}=54.2 \min (2 R, 4 R), \mathrm{t}_{\mathrm{R}}=78.1 \min (2 S, 4 S)$.

\section{4-Butoxy-2-phenyl-1,2,3,4-tetrahydroquinolin-8-ol}<smiles>CCCCOC1C[C@H](c2ccccc2)Nc2c(O)cccc21</smiles>

$\mathrm{R}_{\mathrm{f}} 0.4$ (Hexane: Ethyl acetate=4:1); amorphous solid; $[\alpha]_{\mathrm{D}}{ }^{27} 28.2\left(\mathrm{c} 1.0, \mathrm{CHCl}_{3}\right)(96 \%$ ee); IR $\left(\mathrm{CHCl}_{3}\right) 3412,2961,2359,1512,1460,1238,1219,1067,1024 \mathrm{~cm}^{-1} ;{ }^{1} \mathrm{H}$ $\operatorname{NMR}\left(400 \mathrm{MHz}, \mathrm{CDCl}_{3}\right) \delta=7.47-7.43(\mathrm{~m}, 2 \mathrm{H}), 7.38-7.26(\mathrm{~m}, 3 \mathrm{H}), 7.02(\mathrm{~d}, 1 \mathrm{H}, J=7.3$ $\mathrm{Hz}), 6.60-6.50(\mathrm{~m}, 2 \mathrm{H}), 5.27$ (brs, 1H), 4.85 (dd, 1H, J=10.8, $5.7 \mathrm{~Hz}), 4.48$ (d, 1H, $J=9.9 \mathrm{~Hz}), 4.24$ (brs, $1 \mathrm{H}), 3.67-3.60(\mathrm{~m}, 1 \mathrm{H}), 3.54-3.46(\mathrm{~m}, 1 \mathrm{H}), 2.43-2.37(\mathrm{~m}, 1 \mathrm{H})$, 2.09 (ddd, $1 \mathrm{H}, J=10.8,10.8,10.8 \mathrm{~Hz}), 1.68-1.53$ (m, 2H), 1.47-1.35 (m, 2H), 0.91 (t, 3H, $J=7.51 \mathrm{~Hz}) ;{ }^{13} \mathrm{C} \mathrm{NMR}\left(75 \mathrm{MHz}, \mathrm{CDCl}_{3}\right) \delta=143.6,142.0,134.0,128.6,127.7,126.68$, 123.6, 119.5, 116.9, 113.6, 74.3, 67.9, 55.7, 36.9, 32.2, 19.4, 13.9; Found: C, 76.89; H, 7.89; N, 4.53\%. Calcd for $\mathrm{C}_{19} \mathrm{H}_{23} \mathrm{NO}_{2}: \mathrm{C}, 76.73 ; \mathrm{H}, 7.80 ; \mathrm{N}, 4.71 \%$; HPLC: Daicel Chiralpak AD-H, Hexane/EtOH=20/1, Flow rate $=0.7 \mathrm{~mL} / \mathrm{min}, \mathrm{UV}=254 \mathrm{~nm}, \mathrm{t}_{\mathrm{R}}=16.9$ $\min (2 R, 4 R), \mathrm{t}_{\mathrm{R}}=39.1 \min (2 S, 4 S)$.

\section{4-Butoxy-2-(4-bromophenyl)-1,2,3,4-tetrahydroquinolin-8-ol}<smiles>CCCCO[C@H]1C[C@@H](c2ccc(Br)cc2)Nc2c(O)cccc21</smiles>

$\mathrm{R}_{\mathrm{f}} 0.4$ (Hexane: Ethyl acetate $=4: 1$ ); amorphous solid; $[\alpha]_{\mathrm{D}}{ }^{26} 32.3\left(\mathrm{c} 1.0, \mathrm{CHCl}_{3}\right.$ ) $(89 \%$ ee $($ cis/trans $=96 / 4)) ; \quad$ IR $\left(\mathrm{CHCl}_{3}\right)$ 3595, 3340, 3020, 2961, 2935, 2876, 1620, $1591,1435,1331,1269,1105,1009 \mathrm{~cm}^{-1} ;{ }^{1} \mathrm{H}$ NMR $\left(400 \mathrm{MHz}, \mathrm{CDCl}_{3}\right) \delta=7.48-7.40$ $(\mathrm{m}, 2 \mathrm{H}), 7.35-7.24(\mathrm{~m}, 2 \mathrm{H}), 7.03-6.95(\mathrm{~m}, 1 \mathrm{H}), 6.63-6.48(\mathrm{~m}, 2 \mathrm{H}), 5.35$ (brs, $1 \mathrm{H})$, 4.82-4.79 (m, 1H), 4.50-4.37 (m, 1H), 4.21 (brs, $1 \mathrm{H}), 3.63-3.55(\mathrm{~m}, 1 \mathrm{H}), 3.53-3.46(\mathrm{~m}$, 1H), 2.37-2.33 (m, 1H), 2.06 (ddd, 1H, $J=11.3,11.3,11.3 \mathrm{~Hz}), 1.65-1.33$ (m, 4H), 0.91 $(\mathrm{t}, 3 \mathrm{H}, J=7.4 \mathrm{~Hz}) ;{ }^{13} \mathrm{C}$ NMR $\left(75 \mathrm{MHz}, \mathrm{CDCl}_{3}\right) \delta=142.7,142.1,133.6,131.7,128.4$, 
$123.5,121.3,119.3,119.7,117.1,113.7,74.0,68.0,55.0,36.8,32.1,19.4,13.9$; Found: C, 60.72; H, 5.78; N, 3.55\%. Calcd for $\mathrm{C}_{19} \mathrm{H}_{22} \mathrm{BrNO}_{2}: \mathrm{C}, 60.65 ; \mathrm{H}, 5.89 ; \mathrm{N}$, 3.72\%. HPLC: Daicel Chiralcel OJ-H, Hexane $/ \mathrm{EtOH}=9 / 1$, Flow rate $=0.5 \mathrm{~mL} / \mathrm{min}$, $\mathrm{UV}=254 \mathrm{~nm}, \mathrm{t}_{\mathrm{R}}=44.9 \min (2 R, 4 R), \mathrm{t}_{\mathrm{R}}=33.7 \min (2 S, 4 S)$.

\section{4-Butoxy-2-(2-naphthyl)-1,2,3,4-tetrahydroquinolin-8-ol}<smiles>CCCCOC1C[C@@H](c2ccc3ccccc3c2)Nc2c(O)cccc21</smiles>

$\mathrm{R}_{\mathrm{f}} 0.4$ (Hexane: Ethyl acetate $=4: 1$ ); amorphous solid; $[\alpha]_{\mathrm{D}}{ }^{25} 34.4\left(\mathrm{c} 1.0, \mathrm{CHCl}_{3}\right)(88 \%$ ee); IR $\left(\mathrm{CHCl}_{3}\right)$ 3398, 3300, 3059, 3007, 2961, 2935, 2870, 1620, 1591, 1497, 1435, $1331,1269,1242,1151,1103,1072,1053 \mathrm{~cm}^{-1} ;{ }^{1} \mathrm{H}$ NMR $\left(400 \mathrm{MHz}, \mathrm{CDCl}_{3}\right) \delta=7.91$ (s, 1H), 7.88-7.75 (m, 3H), $7.53(\mathrm{~d}, 1 \mathrm{H}, J=8.4 \mathrm{~Hz}), 7.47-7.40(\mathrm{~m}, 2 \mathrm{H}), 7.10-6.95(\mathrm{~m}$, $1 \mathrm{H}), 6.68-6.42$ (m, 2H), 5.29 (brs, $1 \mathrm{H}), 4.93-4.83$ (m, 1H), 4.65-4.54 (m, 1H), 4.60 (brs, $1 \mathrm{H}), 3.65-3.56(\mathrm{~m}, 1 \mathrm{H}), 3.55-3.47(\mathrm{~m}, 1 \mathrm{H}), 2.46-2.44(\mathrm{~m}, 1 \mathrm{H}), 2.17$ (ddd, $1 \mathrm{H}, J=11.5$, 11.5, $11.5 \mathrm{~Hz}), 1.65-1.48$ (m, 2H), 1.47-1.34 (m, 2H), 0.89 (t, 3H, $J=7.3 \mathrm{~Hz}) ;{ }^{13} \mathrm{C} \mathrm{NMR}$ $\left(75 \mathrm{MHz}, \mathrm{CDCl}_{3}\right) \delta=142.0,141.0,134.0,133.4,133.1,128.4,128.4,127.8,127.6$, 162.1, 125.8, 125.2, 124.8, 123.6, 119.5, 117.0, 113.7, 74.3, 67.9, 55.8, 36.8, 32.2, 19.4, 13.9; Found: C, 79.30; H, 7.49; N, 3.94\%. Calcd for $\mathrm{C}_{23} \mathrm{H}_{25} \mathrm{NO}_{2}$ : C, 79.51; H, 7.25; N, 4.03\%; HPLC: Daicel Chiralcel OJ-H, Hexane/EtOH= 5/1, Flow rate $=0.4 \mathrm{~mL} / \mathrm{min}$, $\mathrm{UV}=254 \mathrm{~nm}, \mathrm{t}_{\mathrm{R}}=46.3 \min (2 R, 4 R), \mathrm{t}_{\mathrm{R}}=35.0 \mathrm{~min}(2 S, 4 S)$.

\section{4-Benzyloxy-2-phenyl-1,2,3,4-tetrahydroquinolin-8-ol}<smiles>Oc1cccc2c1N[C@H](c1ccccc1)C[C@H]2OCc1ccccc1</smiles>

$\mathrm{R}_{\mathrm{f}} 0.4$ (Hexane: Ethyl acetate $=4: 1$ ); amorphous solid; $[\alpha]_{\mathrm{D}}{ }^{26} 18.2\left(\mathrm{c} 1.1, \mathrm{CHCl}_{3}\right.$ ) (91\% ee); $\operatorname{IR}\left(\mathrm{CHCl}_{3}\right)$ 3418, 3009, 2837, 2353, 1512, 1456, 1240, 1217, $1180 \mathrm{~cm}^{-1}$; ${ }^{1} \mathrm{H}$ NMR $\left(400 \mathrm{MHz}, \mathrm{CDCl}_{3}\right) \delta=7.48-7.43(\mathrm{~m}, 2 \mathrm{H}), 7.38-7.22(\mathrm{~m}, 8 \mathrm{H}), 7.08(\mathrm{brd}, 1 \mathrm{H}$, $J=7.5 \mathrm{~Hz}$ ), 6.58 (brdd, $1 \mathrm{H}, J=7.5,7.5 \mathrm{~Hz}), 6.53$ (brd, 1H, J=7.5 Hz), 5.12 (brs, 1H), 4.97 (dd, 1H, $J=10.4,5.7 \mathrm{~Hz}), 4.69$ and $4.58(\mathrm{ABq}, 2 \mathrm{H}, J=11.7 \mathrm{~Hz}), 4.48(\mathrm{brd}, 1 \mathrm{H}, J=11.4$ 
$\mathrm{Hz}$ ), 4.27 (brs, 1H), 2.48-2.43 (m, 1H), 2.19 (ddd, 1H, $J=11.7,11.4,10.4 \mathrm{~Hz}) ;{ }^{13} \mathrm{C}$ $\operatorname{NMR}\left(75 \mathrm{MHz}, \mathrm{CDCl}_{3}\right) \delta=143.5,142.0,138.5,134.1,128.7,128.4,127.7,127.6$, 127.0, 126.7, 123.3, 119.8, 116.9, 113.7, 73.6, 55.6, 36.6; Found: C, 79.97; H, 6.48; N, 4.24\%. Calcd for $\mathrm{C}_{22} \mathrm{H}_{21} \mathrm{NO}_{2}$ : C, 79.73; H, 6.39; N, 4.23\%; HPLC: Daicel Chiralpak AD-H Hexane/EtOH $=20 / 1$ Flow rate $=0.5 \mathrm{~mL} / \mathrm{min}, \mathrm{UV}=317 \mathrm{~nm}, \mathrm{t}_{\mathrm{R}}=49.5$ $\min (2 R, 4 R), \mathrm{t}_{\mathrm{R}}=91.4 \min (2 S, 4 S)$.

\section{4-Phenyl-2,3,3a,4,5,9b-hexahydro-furo[3,2-c]quinolin-6-ol (5a) ${ }^{1}$}<smiles>Oc1cccc2c1N[C@H](c1ccccc1)[C@@H]1CCOC21</smiles>

$\mathrm{R}_{\mathrm{f}} 0.4$ (Hexane: Ethyl acetate $=4: 1$ ); amorphous solid; $[\alpha]_{\mathrm{D}}^{25} 59.0\left(\mathrm{c} 1.0, \mathrm{CHCl}_{3}\right.$ ) (90\% ee); IR $\left(\mathrm{CHCl}_{3}\right)$ 3595, 3385, 3275, 3007, 2922, 2876, 1620, 1593, 1489, 1479, $1450,1434,1365,1340,1298,1256,1180,1155,1063,1047 \mathrm{~cm}^{-1} ;{ }^{1} \mathrm{H}$ NMR (400 $\left.\mathrm{MHz} \mathrm{CDCl}_{3}\right) \quad \delta=7.50(\mathrm{~d}, 2 \mathrm{H}, J=7.9 \mathrm{~Hz}), 7.40-7.27(\mathrm{~m}, 3 \mathrm{H}), 6.97(\mathrm{~d}, 1 \mathrm{H}, J=7.6 \mathrm{~Hz})$, 6.65 (dd, 1H, J=7.6, 7.6 Hz), 6.61 (d, 1H, J=7.6 Hz), 5.69 (brs, 1H), 5.33 (d, 1H, $J=8.1$ $\mathrm{Hz}), 4.66$ (s, 1H), 4.34 (brs, 1H), 3.88-3.82 (m, 1H), 3.76 (ddd, 1H, J=8.3, 8.3, 8.3 Hz), 2.85-2.76 (m, 1H), 2.28-2.22 (m, 1H), 1.58-1.49 (m, 1H); $\left.{ }^{13} \mathrm{C} \mathrm{NMR} \mathrm{(75} \mathrm{MHz,} \mathrm{CDCl}_{3}\right)$ $\delta=142.4,142.2,134.3,128.6,128.6,127.6,126.6,123.3,122.0,118.1,113.3,76.1$, 66.9, 57.2, 45.8, 24.5; Found: C, 76.46; $\mathrm{H}, 6.44 ; \mathrm{N}, 5.18 \%$. Calcd for $\mathrm{C}_{17} \mathrm{H}_{17} \mathrm{NO}_{2}$ : C, 76.38; H, 6.41; N, 5.24\%; HPLC: Daicel Chiralcel OD-H Hexane/ $i$-PrOH=10/1 Flow rate $=0.5 \mathrm{~mL} / \mathrm{min}, \mathrm{UV}=254 \mathrm{~nm}, \mathrm{t}_{\mathrm{R}}=34.0 \mathrm{~min}(2 R, 4 R), \mathrm{t}_{\mathrm{R}}=26.4 \mathrm{~min}(2 S, 4 S)$.

\section{4-(4-Bromophenyl)-2,3,3a,4,5,9b-hexahydro-furo[3,2-c]quinolin-6-ol (5c)}<smiles>Oc1cccc2c1N[C@H](c1ccc(Br)cc1)[C@H]1CCO[C@H]21</smiles>

$\mathrm{R}_{\mathrm{f}} 0.2$ (Hexane: Ethyl acetate=5:1); m.p. $223.0-225.0{ }^{\circ} \mathrm{C} .(\mathrm{EtOH}) ;[\alpha]_{\mathrm{D}}{ }^{28} 63.2$ (c 0.5 , $\left.\mathrm{CHCl}_{3}\right)\left(92 \%\right.$ ee); IR $\left(\mathrm{CHCl}_{3}\right)$ 3393, 3204, 2974, 2935, 2881, 1620, 1587, 1506, 1487 , 1445, 1404, 1369, 1298, 1274, 1248, 1204, 1072, 1031, $1001 \mathrm{~cm}^{-1} ;{ }^{1} \mathrm{H}$ NMR (400 MHz, $\left.\mathrm{CDCl}_{3}\right) \delta=7.51(\mathrm{~d}, 2 \mathrm{H}, J=8.5 \mathrm{~Hz}), 7.39(\mathrm{~d}, 2 \mathrm{H}, J=8.5 \mathrm{~Hz}), 6.99(\mathrm{~d}, 1 \mathrm{H}, J=7.7 \mathrm{~Hz})$, 
$6.68(\mathrm{dd}, 1 \mathrm{H}, J=7.7,7.7 \mathrm{~Hz}), 6.63(\mathrm{~d}, 1 \mathrm{H}, J=7.7 \mathrm{~Hz}), 5.29$ (d, $2 \mathrm{H}, J=7.7 \mathrm{~Hz}), 4.77$ (brs, $1 \mathrm{H}), 4.64$ (brs, $1 \mathrm{H}), 4.25$ (brs, $1 \mathrm{H}), 3.84-3.80$ (m, $1 \mathrm{H}), 3.76-3.70$ (m, $1 \mathrm{H}), 2.77-2.75$ $(\mathrm{m}, 1 \mathrm{H}), 2.18-2.15(\mathrm{~m}, 1 \mathrm{H}), 1.53-1.51(\mathrm{~m}, 1 \mathrm{H}) ;{ }^{13} \mathrm{C} \mathrm{NMR}\left(75 \mathrm{MHz}, \mathrm{CDCl}_{3}\right) \delta=$ 142.1, 141.3, 133.9, 131.7, 128.3, 123.6, 122.2, 121.3, 118.4, 113.3, 75.8, 66.8, 56.7, 45.6, 24.5; Found: C, 58.73; H, 4.55; N, 4.00\%. Calcd for $\mathrm{C}_{17} \mathrm{H}_{16} \mathrm{BrNO}_{2}$ : C, 58.97; H, 4.66; N, 4.05\%; HPLC: Daicel Chiralcel OD-H, Hexane/EtOH=10/1, Flow rate=0.5 $\mathrm{mL} / \mathrm{min}, \mathrm{UV}=254 \mathrm{~nm}, \mathrm{t}_{\mathrm{R}}=22.7 \min (2 R, 4 R), \mathrm{t}_{\mathrm{R}}=31.7 \min (2 S, 4 S)$.

\section{5-Phenyl-3,4,4a,5,6,10b-hexahydro-2H-pyrano[3,2-c]quinolin-7-ol (5b) ${ }^{1}$}<smiles>Oc1cccc2c1N[C@H](c1ccccc1)[C@@H]1CCCO[C@H]21</smiles>

$\mathrm{R}_{\mathrm{f}} 0.4$ (Hexane: Ethyl acetate $\left.=4: 1\right)$; amorphous solid; $[\alpha]_{\mathrm{D}}{ }^{26} 65.7$ (c $\left.0.7, \mathrm{CHCl}_{3}\right)(97 \%$ ee); IR $\left(\mathrm{CHCl}_{3}\right) \quad 3601,3406,3308,2947,2862,1730,1616,1591,1497,1454,1437$, $1375,1346,1279,1261,1180,1155,1090,1070,1038,1015 \mathrm{~cm}^{-1} ;{ }^{1} \mathrm{H}$ NMR (400 $\left.\mathrm{MHz}, \mathrm{CDCl}_{3}\right) \quad \delta=7.47(\mathrm{~d}, 2 \mathrm{H}, J=7.1 \mathrm{~Hz}), 7.38(\mathrm{dd}, 2 \mathrm{H}, J=7.1,7.1 \mathrm{~Hz}), 7.31(\mathrm{dd}, 1 \mathrm{H}$, $J=7.1,7.1 \mathrm{~Hz}$ ), 7.09-7.04 (m, 1H), 6.20-6.12 (m, 2H), 5.37 (d, 1H, J=5.3 Hz), 5.03 (brs, $1 \mathrm{H}), 4.68(\mathrm{~s}, 1 \mathrm{H}), 4.24$ (brs, $1 \mathrm{H}), 3.65-3.58(\mathrm{~m}, 1 \mathrm{H}), 3.49-3.42(\mathrm{~m}, 1 \mathrm{H}), 2.23-2.14(\mathrm{~m}$, 1H), 1.68-1.30 (m, 4H); ${ }^{13} \mathrm{C}$ NMR (75 MHz, $\left.\mathrm{CDCl}_{3}\right) \delta=142.2,141.2,134.3,128.3$, $127.5,126.9,121.0,119.8,117.2,113.2,73.0,60.9,59.1,38.9,25.4,18.4$; Found: C, 76.87; H, 6.82; N, 4.75\%. Calcd for $\mathrm{C}_{18} \mathrm{H}_{19} \mathrm{NO}_{2}$ : C, 76.84; H, 6.81; N, 4.98\%; HPLC: Daicel Chiralpak AD-H Hexane/EtOH=10/1 Flow rate $=0.5 \mathrm{~mL} / \mathrm{min}, \mathrm{UV}=254$ $\mathrm{nm}, \mathrm{t}_{\mathrm{R}}=27.1 \min (2 R, 4 R), \mathrm{t}_{\mathrm{R}}=23.1 \min (2 S, 4 S)$.

\section{6-Benzyloxy-4-phenyl-5,6-dihydro-4H-oxazolo[5,4,3-ij]quinolin-2-one}<smiles>O=c1oc2cccc3c2n1[C@H](c1ccccc1)C[C@H]3OCc1ccccc1</smiles>

To a solution of 4-benzyloxy-2-phenyl-1,2,3,4-tetrahydroquinolin-8-ol (134.2 mg, 0.40 $\mathrm{mmol})$, triphosgene $(54.9 \mathrm{mg}, 0.20 \mathrm{mmol})$ in $\mathrm{CH}_{2} \mathrm{Cl}_{2}(8.0 \mathrm{~mL})$ was added triethylamine 
(122 $\mu \mathrm{L}, 0.88 \mathrm{mmol}$ ) at room temperature. After being stirred at the temperature for $19 \mathrm{~h}$, the mixture was quenched by addition of sat. $\mathrm{NaHCO}_{3}$ solution. The mixture was extracted with ethyl acetate. The combined organic layers were successively washed with $10 \%$ aq. $\mathrm{HCl}$, sat. $\mathrm{NaHCO}_{3}$, brine, dried over anhydrous $\mathrm{Na}_{2} \mathrm{SO}_{4}$, and concentrated to dryness. The crude product was purified by preparative TLC on silica gel $($ hexane $/ \mathrm{EtOAc}=4 / 1)$ to give the product $(126 \mathrm{mg}, 0.35 \mathrm{mmol})$ in $88 \%$ yield .

$\mathrm{R}_{\mathrm{f}} 0.5$ (Hexane: Ethyl acetate $=4: 1$ ); amorphous solid; $[\alpha]_{\mathrm{D}}{ }^{17} 152.4$ (c 1.0, $\mathrm{CHCl}_{3}$ ); IR $\left(\mathrm{CHCl}_{3}\right)$ 3034, 2926, 2856, 1769, 1660, 1620, 1493, 1477, 1396, 1358, 1254, 1238, $1034,1007 \mathrm{~cm}^{-1} . \quad{ }^{1} \mathrm{H}$ NMR $\left(400 \mathrm{MHz}, \mathrm{CDCl}_{3}\right) \delta=7.34-7.07(\mathrm{~m}, 11 \mathrm{H}), 6.93-6.86(\mathrm{~m}$, $2 \mathrm{H}), 5.43-5.37(\mathrm{~m}, 1 \mathrm{H}), 4.79-4.74(\mathrm{~m}, 1 \mathrm{H}), 4.41$ and $4.35(\mathrm{ABq}, 2 \mathrm{H}, J=11.5 \mathrm{~Hz})$, 2.86-2.76 (m, 1H), 2.50-2.40 (m, 1H); ${ }^{13} \mathrm{C} \mathrm{NMR}\left(75 \mathrm{MHz}, \mathrm{CDCl}_{3}\right) \delta=153.0,141.8$, $138.7,137.5,128.9,128.7,128.2,127.6,127.5,127.2,125.8,122.4,121.9,110.0,109.6$, 70.3, 69.8, 53.0, 35.5; Found: $\mathrm{C}, 77.10 ; \mathrm{H}, 5.42 ; \mathrm{N}, 3.82 \%$. Calcd for $\mathrm{C}_{23} \mathrm{H}_{19} \mathrm{NO}_{3}$ : C, $77.29 ; \mathrm{H}, 5.36 ; \mathrm{N}, 3.92 \%$.

\section{6-Ethoxy-4-phenyl-5,6-dihydro-4H-oxazolo[5,4,3-ij]quinolin-2-one}<smiles>CCOC1C[C@H](c2ccccc2)n2c(=O)oc3cccc1c32</smiles>

$\mathrm{R}_{\mathrm{f}} 0.5$ (Hexane: Ethyl acetate $\left.=4: 1\right)$; mp 155.0-158.0 ${ }^{\circ} \mathrm{C}\left(\mathrm{CH}_{2} \mathrm{Cl}_{2}\right) ;[\alpha]_{\mathrm{D}}{ }^{20} 116.9(\mathrm{c} 1.0$, $\left.\mathrm{CHCl}_{3}\right)$; IR $\left(\mathrm{CHCl}_{3}\right)$ 3040, 2928, 2870, 2359, 1778, 1664, 1616, 1487, 1352, 1333, 1269, 1123, 1082, $1024 \mathrm{~cm}^{-1} ; \quad{ }^{1} \mathrm{H}$ NMR $\left(400 \mathrm{MHz}, \mathrm{CDCl}_{3}\right) \delta=7.35-7.07(\mathrm{~m}, 8 \mathrm{H}), 5.33$ (dd, $1 \mathrm{H}, J=5.1,5.1 \mathrm{~Hz}), 4.66(\mathrm{dd}, 1 \mathrm{H}, J=4.2,4.0 \mathrm{~Hz}), 3.41-3.25(\mathrm{~m}, 2 \mathrm{H}), 2.71-2.64(\mathrm{~m}$, $1 \mathrm{H}), 2.41-2.33(\mathrm{~m}, 1 \mathrm{H}), 0.85(\mathrm{t}, 3 \mathrm{H}, J=7.0 \mathrm{~Hz}) .{ }^{13} \mathrm{C} \mathrm{NMR}\left(75 \mathrm{MHz}, \mathrm{CDCl}_{3}\right) \delta=152.9$, 141.6, 138.6, 128.8, 128.4, 127.5, 125.8, 122.1, 121.9, 120.5, 109.3, 70.2, 63.9, 53.3, 35.2, 14.8; Found: C, 73.54; H, 5.89; N, 4.54\%. Calcd for $\mathrm{C}_{18} \mathrm{H}_{17} \mathrm{NO}_{3}$ : C, 73.20; $\mathrm{H}$, $5.80 ; \mathrm{N}, 4.74 \%$.

\section{6-Hydroxy-4-phenyl-5,6-dihydro-4H-oxazolo[5,4,3-ij]quinolin-2-one}


<smiles>O=c1oc2cccc3c2n1[C@H](c1ccccc1)C[C@H]3O</smiles>

A solution of 6-benzyloxy-4-phenyl-5,6-dihydro-4H-oxazolo[5,4,3-ij]quinolin-2-one (110.2 mg, $0.31 \mathrm{mmol})$, Pd-C (59 mg) in $\mathrm{AcOH}(4.0 \mathrm{~mL})$ was allowed to stirred in $\mathrm{H}_{2}$ atmosphere for $64 \mathrm{~h}$. The mixture was filtered over Celite to remove the catalyst, and the catalyst was washed with ethyl acetate. The filtrate was concentrated and the crude mixture was purified by preparative TLC on silica gel (hexane: ethyl acetate 1:1) to give the title compound $(84.7 \mathrm{mg}, 0.31 \mathrm{mmol})$ in $98 \%$ yield.

$\mathrm{R}_{\mathrm{f}} 0.3$ (Hexane: Ethyl acetate $\left.=1: 1\right) ; \quad \operatorname{mp} 72.0-75.0{ }^{\circ} \mathrm{C}\left(\mathrm{CH}_{2} \mathrm{Cl}_{2}\right) ; \quad[\alpha]_{\mathrm{D}}{ }^{18} 137.9(\mathrm{c} 0.6$, $\left.\mathrm{CHCl}_{3}\right)$; IR $\left(\mathrm{CHCl}_{3}\right)$ 3585, 3466, 3030, 3007, 2918, 1782, 2663, 1618, 1277, 1393, $1377,1369,1256,1103,1045,1022 \mathrm{~cm}^{-1} ;{ }^{1} \mathrm{H}$ NMR $\left(400 \mathrm{MHz}, \mathrm{CDCl}_{3}\right) \delta=7.34-7.08$ (m, 8H), 5.29 (t, 1H, J=6.0 Hz), 4.97 (brs, 1H), 2.55-2.43 (m, 2H), 2.09 (brs, $1 \mathrm{H}) ; \quad{ }^{13} \mathrm{C}$ $\operatorname{NMR}\left(75 \mathrm{MHz}, \mathrm{CDCl}_{3}\right) \delta=152.8,141.4,137.9,129.0,128.3,128.1,128.1,125.6$, 122.5, 122.4, 121.3, 109.2, 63.8, 53.5, 39.9; Found: C, 71.73; H, 5.17; N, 5.07\%. Calcd for $\mathrm{C}_{16} \mathrm{H}_{13} \mathrm{NO}_{3}$ : C, 71.90; H, 4.90; N, 5.24\%.

\section{4-Phenyl-5,6-dihydro-4H-oxazolo[5,4,3-ij]quinolin-2-one}<smiles>O=c1oc2cccc3c2n1[C@H](c1ccccc1)CC3</smiles>

A solution of 6-ethoxy-4-phenyl-5,6-dihydro-4H-oxazolo[5,4,3-ij]quinolin-2-one (34.7 $\mathrm{mg}, 0.13 \mathrm{mmol}), \mathrm{Pd}(\mathrm{OH})_{2}(29 \mathrm{mg})$ in $\mathrm{AcOH}(4.0 \mathrm{~mL})$ was allowed to stirred in $\mathrm{H}_{2}$ atmosphere for $37 \mathrm{~h}$. The mixture was filtered over Celite to remove the catalyst, and the catalyst was washed with ethyl acetate. The filtrate was concentrated and the crude mixture was purified by preparative TLC on silica gel (hexane: ethyl acetate 1:1) to give the title compound (37.0 $\mathrm{mg}, 0.11 \mathrm{mmol})$ in $83 \%$.

$\mathrm{R}_{\mathrm{f}} 0.3$ (Hexane: Ethyl acetate $\left.=4: 1\right) ; \quad \mathrm{mp} 118.0-120.0{ }^{\circ} \mathrm{C}\left(\mathrm{CH}_{2} \mathrm{Cl}_{2}\right) ; \quad[\alpha]_{\mathrm{D}}{ }^{27} 126.7(\mathrm{c}$ 
$\left.0.8, \mathrm{CHCl}_{3}\right)(94 \%$ ee $) ; \quad$ IR $\left(\mathrm{CHCl}_{3}\right) 2935,1773,1711,1663,1616,1493,1477,1454$, 1398, 1360, 1337, 1261, 1217, 1038, $1009 \mathrm{~cm}^{-1}$. ${ }^{1} \mathrm{H}$ NMR $\left(400 \mathrm{MHz}, \mathrm{CDCl}_{3}\right) \delta=$ 7.36-7.25 (m, 3H), 7.12-7.05 (m, 4H), 6.99 (d, 1H, J=6.8 Hz), 5.42 (dd, 1H, J=7.7, 4.2 $\mathrm{Hz}), 2.78-2.70(\mathrm{~m}, 1 \mathrm{H}), 2.64-2.54(\mathrm{~m}, 1 \mathrm{H}), 2.37-2.23(\mathrm{~m}, 2 \mathrm{H}) ;{ }^{13} \mathrm{C}$ NMR $(75 \mathrm{MHz}$, $\left.\mathrm{CDCl}_{3}\right) \delta=153.3,141.4,139.1,128.8,128.6,127.9,125.5,122.0,121.9,120.4,107.4$, 53.3, 29.8, 19.5; Found: C, 76.36; $\mathrm{H}, 5.74 ; \mathrm{N}, 5.49 \%$. Calcd for $\mathrm{C}_{16} \mathrm{H}_{13} \mathrm{NO}_{2}$ : C, 76.48; H, 5.21; N, 5.57\%; HPLC: Daicel Chiralpak AD-H Hexane $/ i-\mathrm{PrOH}=30 / 1$ Flow rate $=1.0 \mathrm{~mL} / \mathrm{min}, \mathrm{UV}=233 \mathrm{~nm}, \mathrm{t}_{\mathrm{R}}=22.4 \min (2 R), \mathrm{t}_{\mathrm{R}}=23.8 \mathrm{~min}(2 S)$.

\section{4-Phenyl-4,5-dihydrooxazolo[5,4,3-ij]quinolin-2,6-dione}<smiles>O=C1C[C@@H](c2ccccc2)n2c(=O)oc3cccc1c32</smiles>

A solution of 6-hydroxy-4-phenyl-5,6-dihydro-4H-oxazolo[5,4,3-ij]quinolin-2-one (82.5 mg, $0.31 \mathrm{mmol})$ in $\mathrm{CH}_{2} \mathrm{Cl}_{2}$ was treated with $\mathrm{MnO}_{2}(1.44 \mathrm{~g}$ ) at room temperature for $40 \mathrm{~min}$. The mixture was filtered over Celite to remove the solids, and the solids was washed with ethyl acetate. The filtrate was concentrated and the crude mixture was purified by preparative TLC on silica gel (hexane: ethyl acetate 4:1) to give the title compound (15.6 mg, $0.17 \mathrm{mmol}$ ) in 56\%.

$\mathrm{R}_{\mathrm{f}} 0.2$ (Hexane: Ethyl acetate $\left.=4: 1\right) ; \quad \mathrm{mp} 121.0-122.0{ }^{\circ} \mathrm{C}\left(\mathrm{CH}_{2} \mathrm{Cl}_{2}\right) ; \quad[\alpha]_{\mathrm{D}}{ }^{22}-245.9(\mathrm{c}$ 0.7, $\left.\mathrm{CHCl}_{3}\right)\left(90 \%\right.$ ee); IR $\left(\mathrm{CHCl}_{3}\right) 3032,1791,1697,1649,1622,1497,1352,1327$, $1288,1259,1242,1219,1061,1034 \mathrm{~cm}^{-1} ; \quad{ }^{1} \mathrm{H}$ NMR $\left(400 \mathrm{MHz}, \mathrm{CDCl}_{3}\right) \delta=7.59(\mathrm{~d}, 1 \mathrm{H}$, $J=8.1 \mathrm{~Hz}), 7.37$ (d, 1H, J=7.9 Hz), 7.36-7.30 (m, 3H), 7.28-7.22 (m, 2H), 7.18 (dd, 1H, $J=8.1,7.9 \mathrm{~Hz}), 5.67$ (dd, 1H, $J=7.3,3.1 \mathrm{~Hz}), 3.40$ (dd, 1H, $J=16.6,7.3 \mathrm{~Hz}), 3.14$ (dd, $1 \mathrm{H}, J=16.6,3.3 \mathrm{~Hz}) ;{ }^{13} \mathrm{C} \mathrm{NMR}\left(75 \mathrm{MHz}, \mathrm{CDCl}_{3}\right) \delta=189.0,152.2,141.7,137.5$, 135.6, 129.3, 129.0, 126.1, 122.4, 119.4, 116.6, 115.0, 55.5, 44.1; Found: C, 72.67; H, 4.14; N, 5.04\%. Calcd for $\mathrm{C}_{16} \mathrm{H}_{11} \mathrm{NO}_{3}: \mathrm{C}, 72.45 ; \mathrm{H}, 4.18 ; \mathrm{N}, 5.28 \%$; HPLC: Daicel Chiralpak AD-H Hexane $/ i-\mathrm{PrOH}=30 / 1$ Flow rate $=1.0 \mathrm{~mL} / \mathrm{min}, \mathrm{UV}=254 \mathrm{~nm}, \mathrm{t}_{\mathrm{R}}=40.1$ $\min (2 R), \mathrm{t}_{\mathrm{R}}=48.9 \min (2 S)$. 
Reference

1) Ma, Y.; Qian, C.; Xie, M.; Sun, J. J. Org. Chem. 1999, 64, 6462-6467. 